\title{
Ciclo biológico de Caligo Idomeneus \\ (Nymphalidae) bajo condiciones controladas con jardines verticales en Tarapoto
}

\author{
Biological cycle of Caligo idomeneus (Nymphalidae) \\ Breeding, under controlled conditions with vertical gardens \\ in Tarapoto
}

Doria Bolaños, Manuel Santiago ${ }^{1[0000-0001-9202-973 X]}$, Ramírez Navarro, Manuel ${ }^{1[0000-0002-0822-0249]}$ y Fachín Ruíz, Grecia ${ }^{1[0000-0001-7770-7264]}$

${ }^{1}$ Universidad Nacional de San Martín mdoriadunsm.edu.pe

Resumen. El trabajo fue ejecutado en el mariposario de la UNSM-T, Morales, desarrollando una metodología para la cría de Caligo idomeneus (Lepidoptera: Nymphalidae) bajo condiciones de mariposario, con jardines verticales y sustrato de alimentación con heliconia y plátano. Los huevos tuvieron una duración de 7 días, la larva pasó por 5 estadíos, el primer instar tuvo una duración de 2 a 3 días, no tuvo presencia de cuernos, el promedio de ancho de cápsula cefálica fue de $0,38 \mathrm{~mm}$ y una longitud de $1,9 \mathrm{~mm}$; en el segundo instar se pudo apreciar la aparición de dos pequeños cuernos en la parte anterior de la cabeza de $0,36 \mathrm{~mm}$ en promedio, tuvo un promedio de ancho de cápsula cefálica de $0,56 \mathrm{~mm}$ y duración de 2,2 días y una longitud de 4,12 mm; el tercer instar tuvo una duración de 3 días, con un ancho de cápsula cefálica de $1,04 \mathrm{~mm}$, longitud de cuernos de $5,74 \mathrm{~mm}$ y una longitud de 10,40 mm; el cuarto instar tuvo una duración de 4,4 días y 4,27 mm en promedio de ancho de cápsula cefálica, con una longitud de cuernos de 8,02 mm y una longitud de $18,07 \mathrm{~mm}$; el quinto instar tuvo $6,26 \mathrm{~mm}$ de ancho de cápsula cefálica y tuvo una duración de 8,86 días, con longitud de cuernos de $14,93 \mathrm{~mm}$ y $36,33 \mathrm{~mm}$ de longitud; el promedio de pupa fue de 17,46 días. La duración del ciclo, desde huevo a adulto fue de 66,90 días en promedio.

Citar como: Doria Bolaños , M., Ramirez Navarro, M., \& Fachin Ruiz, G. (2021). Ciclo biológico de Caligo Idomeneus (Nymphalidae) bajo condiciones controladas con jardines verticales en Tarapoto. Revista Agrotecnológica Amazónica, 1(1), 20-33. https://doi.org/10.51252/ra a.v1i1.105

Recibido: $15 / 11 / 2020$

Revisado: $15 / 12 / 2020$

Publicado: 31/01/2021
Palabras clave: caligo, cápsula cefálica, heliconia, instar, mariposario

Abstract. The work was carried out in the butterfly farm of the UNSM-T, Morales, developing a methodology for the breeding of Caligo idomeneus (Lepidoptera: Nymphalidae) under butterfly farm conditions, with vertical gardens and feeding substrate with heliconia and banana. The eggs lasted 7 days, the larva went through 5 stages, the first instar lasted 2 to 3 days, had no horns, the average width of the cephalic capsule was $0.38 \mathrm{~mm}$ and a length $1.9 \mathrm{~mm}$; In the second instar it was possible to see the appearance of two small horns in the anterior part of the head of $0.36 \mathrm{~mm}$ on average, it had an average width of the cephalic capsule of $0.56 \mathrm{~mm}$ and duration of 2.2 days and a length $4.12 \mathrm{~mm}$; the third instar lasted 3 days, with a head capsule width of $1.04 \mathrm{~mm}$, horn length of $5.74 \mathrm{~mm}$, and a length of $10.40 \mathrm{~mm}$; the fourth instar had a duration of 4.4 days and an average width of the cephalic capsule $4.27 \mathrm{~mm}$, with a horn length of $8.02 \mathrm{~mm}$ and a length of $18.07 \mathrm{~mm}$; the fifth instar had a head capsule width of $6.26 \mathrm{~mm}$ and a duration of 8.86 days, with a horn length of $14.93 \mathrm{~mm}$ and a length of $36.33 \mathrm{~mm}$; the average pupal period was 17.46 days. The duration of the cycle, from egg to adult, was 66.90 days on average.

Keywords: butterfly house, caligo, cephalic capsule, heliconia, instar 


\section{Introducción}

Los insectos habitan la tierra desde tiempos muy remotos. Se han encontrado fósiles que datan de del periodo carbonífero superior de la era Paleozoica, es decir unos 250 a 300 millones de años de antigüedad. La capacidad de reproducción de los insectos es extraordinariamente grande. El número de huevos que deposita a cada hembra y el corto ciclo de desarrollo que muchas veces presentan, determinar que el número potencial de descendientes alcance características espectaculares en un corto plazo (Zapata, 1986).

Perú posee una naturaleza muy singular y compleja. Su variada geografía sumada a su diversidad de pisos ecológicos y climas, permite encontrar un mosaico de ecosistemas distribuidos a lo largo y ancho de su territorio; motivo por el cual es considerado un país megadiverso. (Prompex, IIAP y GTZ, 2007).

Las mariposas forman parte de esta gran riqueza biológica (Barth, 1991); son apreciadas por su gran belleza y su utilidad como bioindicadoras del estado de salud de los ecosistemas naturales (Barth, 1991), lo cual las convierte en un recurso natural de gran potencial que debe ser aprovechado de manera sostenible. La crianza y comercialización de mariposas en el Perú, especialmente la de especies de la Amazonía, podría desarrollarse como una fuente nueva de ingreso para las comunidades locales $\mathrm{y}$, al mismo tiempo, como un aspecto interesante para el Ecoturismo.

La conservación de las mariposas no es una tarea imposible. Su hábitat se ve agredido por las actividades del hombre en la naturaleza, que provocan un retroceso en el número de plantas florales y también en las posibilidades de reproducción y alimentación de las mariposas, en tanto este trabajo de investigación permitió desarrollar un protocolo de crianza de una especie de mariposa para así poder contribuir hacia un manejo sustentable de la biodiversidad.

Los objetivos del trabajo de investigación fueron obtener un protocolo para la crianza intensiva de la mariposa, así como evaluar el ciclo biológico bajo condiciones controladas, determinar la preferencia alimenticia y, la viabilidad y sostenibilidad de cría de Caligo idomeneus (Lepidoptera: Nymphalidae) bajo condiciones controladas.

\section{Materiales y métodos}

\subsection{Ubicación e instalación del zoocriadero (mariposario).}

Se instaló un zoocriadero (mariposario) en la Ciudad Universitaria - UNSM-T, con las instalaciones apropiadas para la reproducción, como jardines verticales con troncos, con riego por goteo y una pileta central. El mariposario fue dotado de las condiciones ambientales requeridas por la mariposa como temperatura, humedad relativa y porcentaje de sombra, protección y agua corriente (Cantarero, Canales, Mendoza y Martínez, 2012).

Citar como: Doria Bolaños , M., Ramirez Navarro, M., \& Fachin Ruiz, G. (2021). Ciclo biológico de Caligo Idomeneus (Nymphalidae) bajo condiciones controladas con jardines verticales en Tarapoto. Revista Agrotecnológica Amazónica, 1(1), 20-33. https://doi.org/10.51252/raa.v1i1.105 


\subsection{Establecimiento de plantas hospederas.}

\section{a. Identificación de plantas hospederas}

Se recopiló información sobre plantas hospederas en los diferentes grupos de mariposas, así se instaló como planta hospedera a Heliconia caribaea, Heliconia bihai y Musa paradisiaca, dentro del mariposario y fuera de él como plantas de reserva (Constantino, 1996).

\section{b. Manejo de planta hospedera}

Se instaló plantas que sirvieron de alimento para las larvas (De Vries,1987), en el interior del área destinada al zoocriadero (mariposario).

\subsection{Obtención del plantel genético}

Para iniciar la crianza de la mariposa se adquirió un plantel de 10 adultos (machos y hembras), posturas y larvas de diferentes estadios; a partir de estos, se obtuvo el primer plantel genético (f1), que sirvieron para la investigación.

\subsection{Manejo del plantel genético de Caligo idomeneus en laboratorio}

Para alimentar a los adultos fue necesario combinarles fruta fresca con fruta en estado de fermentación (plátano, papaya, naranja, etc.). También se agregó excremento animal, orina y carroña para dar sales a los machos; esto es necesario para que alcancen su madurez reproductiva, metodología planteada por Prompex, IIAP y GTZ (2007). En caso de las larvas colectadas, fueron estabuladas en tapers de crianza con hojas de la panta hospedera hasta lograr la emergencia de los adultos.

\subsection{Crianza de Caligo idomeneus (Prompex, IIAP y GTZ, 2007).}

\section{a. Colecta y manejo de huevos}

Los huevos fueron colectados diariamente y colocados en envases de plástico, rotulados y transportados al Laboratorio de Entomología (Asto y Gutierrez, 2014).

\section{b. Manejo de larvas}

Se verificó que la larva recién eclosionada haya comido el corion, posteriormente se las alimentó con hojas de la planta hospedera. Se individualizó la crianza para ser evaluadas durante todo el ciclo biológico. En los primeros estadios las orugas están dentro de contenedores de plástico pequeños, con la mayor área de la tapa cubierta con una tela de tul para la recirculación de aire. A partir del tercer estadio, las orugas fueron trasladadas a envases más grandes con suficiente cantidad de plantas hospederas.

\section{c. Manejo de pupas}


Una vez transformada en pupa, se procedió a separarlas del lugar donde se encuentra cortando el pedazo de planta hospedera donde se ha fijado, para luego con ayuda de un alfiler, se cuelgue en las cajas de empupamiento.

\section{d. Obtención de adultos en laboratorio}

Una vez obtenidos los adultos fueron liberados en el mariposario para que inicien la postura y, a partir de esas posturas, iniciar el estudio del ciclo biológico dentro del mariposario, tales como cantidad de postura, tiempo de incubación, estadios larvales, pupas, adultos y longevidad de machos y hembras (Flores y Gil, 2012).

\subsection{Técnicas de procedimiento $\mathrm{y}$ análisis de datos}

Instalado el mariposario se procedió a la selección de 10 parejas de adultos dentro del mismo, sobre esta muestra se obtuvieron los datos de los indicadores. La obtención de datos de duración del estado larval, duración del estado pupal, porcentaje de emergencia de adultos, ratio sexual y longevidad de ambos sexos se obtuvieron en el Laboratorio de Entomología. Esta actividad fue repetida por dos generaciones. Los datos se presentan en tablas, con promedios y significancia. La comparación de promedios se realizó aplicando estadística simple no paramétrica.

\section{Resultados y discusiones}

\subsection{Estadios de Caligo idomeneus en días.}

Tabla 1

Duración de los estadios de C. idomeneus en días

\begin{tabular}{lll}
\hline Estadios & \multicolumn{2}{l}{ Duración en días } \\
\hline huevo & 7,0 & $(6$ a 8$)$ \\
Larva I & 2,06 & $(2$ a 3) \\
Larva II & 2,20 & $(2$ a 3$)$ \\
Larva III & 3,00 & $(3)$ \\
Larva IV & 4,40 & $(4$ a 5) \\
Larva V & 8,86 & $(8$ a 10$)$ \\
Pupa & 17,46 & $(15$ a 21$)$ \\
Adulto & 21,92 & $(21$ a 30$)$ \\
total & 66,90 & $(61$ a 63$)$ \\
\hline
\end{tabular}

De la Tabla 1 se deduce los estadios de la mariposa: huevo promedio 7 días, 5 estadios larvales con duración promedio de 20,52 días, pupa con duración promedio de 17,46 días y longevidad de 21,92 días en promedio, total para el ciclo biológico de 66,90 días en promedio (61 a 63 días).

\subsection{Del tamaño de cápsula cefálica, longitud de cuernos y longitud de larvas}

Citar como: Doria Bolaños, M., Ramirez Navarro, M., \& Fachin Ruiz, G. (2021). Ciclo biológico de Caligo Idomeneus (Nymphalidae) bajo condiciones controladas con jardines verticales en Tarapoto. Revista Agrotecnológica Amazónica, 1(1), 20-33. https://doi.org/10.51252/raa.v1i1.105 
Tabla 2

Prueba de Nornalidad para ancho de la cápsula cefálica y longitud de la larva (mm) Estadio I

\begin{tabular}{lrrrrrr}
\hline & \multicolumn{3}{c}{ Kolmogorov-Smirnov } & \multicolumn{3}{c}{ Shapiro-Wilk } \\
\cline { 2 - 7 } & Estadístico & gl & Sig. & Estadístico & gl & Sig. \\
\hline Ancho de la cápsula cefálica & 0,188 & 15 & 0,162 & 0,928 & 15 & 0,25 \\
Longitu de larva & 0,155 & 15 & $0,200^{*}$ & 0,917 & 15 & 0,17 \\
\hline
\end{tabular}

*. Esto es un límite inferior de la significación verdadera.

a. Corrección de significación de Lilliefors

En la Tabla 2, se observa la prueba de normalidad del estadístico Shapiro-Wilk, ya que nuestros datos son menores a 50, en ella se indica que el p. valor o "Sig" es mayor a 0,01 afirmándonos que los datos siguen una distribución normal, permitiéndonos hacer una prueba paramétrica.

Tabla 3

Regresión Lineal Simple para ancho de la cápsula cefálica y longitud de la larva (mm) Estadio I

\begin{tabular}{|c|c|c|c|c|}
\hline Modelo & $\mathbf{R}$ & $\mathbf{R}$ cuadrado & $\begin{array}{l}R \text { cuadrado } \\
\text { ajustado }\end{array}$ & $\begin{array}{c}\text { Error estándar de la } \\
\text { estimación }\end{array}$ \\
\hline 1 & $0,103 \mathrm{a}$ & 0,011 & $-0,066$ & 0,03460 \\
\hline
\end{tabular}

En la Tabla 3, muestra el R2 o Coeficiente de Determinación, que expresa la varianza de la variable dependiente que esta explicada por la variable independiente, en este cuadro se observa que tiene un valor de $1,1 \%$ de la variación de la cápsula cefálica esta explicada por la longitud de larva, así mismo se muestra el Coeficiente de Correlación de Pearson con 10,3\% existiendo una correlación no significativa entre las variables de estudio.

Tabla 4

ANVA de Regresión Lineal Simple para ancho de la cápsula cefálica y longitud de la larva ( $\mathrm{mm})$ Estadio I ANVA $^{\mathrm{a}}$

\begin{tabular}{llcrrrl}
\hline & Modelo & $\begin{array}{c}\text { Suma de } \\
\text { cuadrados }\end{array}$ & gl & $\begin{array}{c}\text { Media } \\
\text { cuadrática }\end{array}$ & F & Sig. \\
\hline 1 & Regresión & 0,000 & 1 & 0,000 & 0,139 & $0,715 \mathrm{~b}$ \\
& Residuo & 0,016 & 13 & 0,001 & & \\
& Total & 0,016 & 14 & & & \\
\hline
\end{tabular}

b. Predictores: (Constante), Longitud de larva

El Tabla 4 se observa que $\mathrm{p}$. valor $>0,05$ que nos indica que no es posible construir un modelo de regresión a partir de estas dos variables, por lo que se concluye que las variables no están linealmente relacionadas. 
Tabla 5

Prueba de Nornalidad para ancho de la cápsula cefálica, longitud de los cuernos y longitud de la larva (mm) Estadio II

\begin{tabular}{|c|c|c|c|c|c|c|}
\hline & \multicolumn{3}{|c|}{ Kolmogorov-Smirnov ${ }^{a}$} & \multicolumn{3}{|c|}{ Shapiro-Wilk } \\
\hline & Estadístico & gl & Sig. & Estadístico & gl & Sig. \\
\hline $\begin{array}{l}\text { Ancho de la } \\
\text { Cefálica }\end{array}$ & 0,254 & 15 & 0,010 & 0,793 & 15 & 0,003 \\
\hline Longitud de cuernos & 0,172 & 15 & $0,200^{*}$ & 0,927 & 15 & 0,244 \\
\hline Longitud de larva & 0,211 & 15 & 0,070 & 0,885 & 15 & 0,056 \\
\hline
\end{tabular}

a. Corrección de significación de Lilliefors

En la Tabla 5, se observa la prueba de normalidad del estadístico Shapiro-Wilk, ya que nuestros datos son menores a 50, en ella se indica que el p. valor o "Sig" es mayor a 0,01 en lo que se refiere a longitud de cuernos y longitud de larvas, afirmándonos que los datos siguen una distribución normal, que nos permite hacer una prueba paramétrica, en cambio el ancho de la cápsula cefálica el p. valor o "Sig" es menor a 0,01 lo cual no se puede hacer una prueba paramétrica.

\section{Tabla 6}

Regresión Lineal Simple para longitud de cuernos y longitud de la larva (mm) Estadio II Resumen del modelo

\begin{tabular}{cccccc}
\hline Modelo & R & R cuadrado & $\begin{array}{c}\text { R cuadrado } \\
\text { ajustado }\end{array}$ & $\begin{array}{c}\text { Error estándar de la } \\
\text { estimación }\end{array}$ \\
\hline 1 & $0,313 \mathrm{a}$ & 0,098 & 0,028 & 0,03701 \\
\hline a. Predictores: (Constante), Longitud de larva & & & & &
\end{tabular}

En la Tabla 6, muestra el R2 o Coeficiente de Determinación, que expresa la varianza de la variable dependiente que esta explicada por la variable independiente, en este cuadro se observa que tiene un valor de 9,8\% de la variación de la longitud del cuerno esta explicada por la longitud de larva, así mismo se muestra el Coeficiente de Correlación de Pearson con 31.3\% existiendo una correlación baja entre las variables de estudio.

Tabla 7

ANVA de Regresión Lineal Simple para longitud del cuerno y longitud de larva (mm) Estadio II ANVA $^{\mathbf{a}}$

\begin{tabular}{|c|c|c|c|c|c|c|}
\hline & Modelo & $\begin{array}{c}\text { Suma de } \\
\text { cuadrados }\end{array}$ & gl & $\begin{array}{c}\text { Media } \\
\text { cuadrática }\end{array}$ & $\mathbf{F}$ & Sig. \\
\hline \multirow[t]{3}{*}{1} & Regresión & 0,002 & 1 & 0,002 & 1,410 & $0,256^{\mathrm{b}}$ \\
\hline & Residuo & 0,018 & 13 & 0,001 & & \\
\hline & Total & 0,020 & 14 & & & \\
\hline
\end{tabular}

En la Tabla 7 se observa que p. valor $>0,05$ lo que indica que no es posible construir un modelo de regresión a partir de estas dos variables, por lo que se concluye que las variables no están linealmente relacionadas. 


\section{Tabla 8}

Prueba de normalidad para ancho de la cápsula cefálica, longitud de los cuernos y longitud de la larva (mm) Estadio III

Pruebas de normalidad

\begin{tabular}{lcccccc}
\hline & \multicolumn{3}{c}{ Kolmogorov-Smirnov $^{\mathbf{a}}$} & \multicolumn{2}{c}{ Shapiro-Wilk } \\
\cline { 2 - 7 } & Estadístico & gl & \multicolumn{1}{c}{ Sig. } & Estadístico & gl & Sig. \\
\hline Ancho de la Cápsula Cefálica & 0,173 & 15 & $0,200^{*}$ & 0,876 & 15 & 0,042 \\
Longitud de cuernos & 0,273 & 15 & 0,004 & 0,839 & 15 & 0,012 \\
Longitud de larva & 0,208 & 15 & 0,079 & 0,903 & 15 & 0,107 \\
\hline
\end{tabular}

*. Esto es un límite inferior de la significación verdadera.

a. Corrección de significación de Lilliefors

En la Tabla 8, se observa la prueba de normalidad del estadístico Shapiro-Wilk, ya que los datos son menores a 50, en ella se indica que el p. valor o "Sig" es mayor a 0,01 en lo que se refiere al ancho de la cápsula cefálica, longitud de cuernos y longitud de larvas, nos confirma que los datos siguen una distribución normal, lo cual os permitirá hacer una prueba paramétrica.

Tabla 9

Prueba de Regresión Lineal Simple para cápsula cefálica, longitud de cuernos y longitud de la larva (mm) Estadio III

\begin{tabular}{|c|c|c|c|c|}
\hline Modelo & $\mathbf{R}$ & $\mathbf{R}$ cuadrado & $\begin{array}{l}R \text { cuadrado } \\
\text { ajustado }\end{array}$ & $\begin{array}{l}\text { Error estándar } \\
\text { de la estimación }\end{array}$ \\
\hline 1 & $0,384 a$ & 0,147 & 0,005 & 0,40396 \\
\hline
\end{tabular}

En la Tabla 9, muestra el R2 o Coeficiente de Determinación, que expresa la varianza de la variable dependiente que esta explicada por la variable independiente, en este cuadro se observa que tiene un valor de $14,7 \%$ de la variación de la longitud del cuerno esta explicada por la longitud de larva, así mismo se muestra el Coeficiente de Correlación de Pearson con 38.4\% existiendo una correlación baja entre las variables en estudio.

\section{Tabla 10}

ANVA de Regresión Lineal Simple para la cápsula cefálica, longitud del cuerno y longitud de la larva (mm) Estadio III

\begin{tabular}{|c|c|c|c|c|c|c|}
\hline & Modelo & Suma de cuadrados & gl & $\begin{array}{c}\text { Media } \\
\text { cuadrática }\end{array}$ & $\mathbf{F}$ & Sig. \\
\hline \multirow[t]{3}{*}{1} & Regresión & 0,338 & 2 & 0,169 & 1,035 & $0,385 b$ \\
\hline & Residuo & 1,958 & 12 & 0,163 & & \\
\hline & Total & 2,296 & 14 & & & \\
\hline
\end{tabular}

En la Tabla 10 se observa que $\mathrm{p}$. valor $>0,05$ lo que indica que no es posible construir un modelo de regresión a partir de estas tres variables, por lo que se concluye que las variables no están linealmente relacionadas. 


\section{Tabla 11}

Prueba de Nornalidad para ancho de la cápsula cefálica, longitud de los cuernos y longitud de la larva (mm) Estadio IV

\begin{tabular}{lcccccc}
\hline & \multicolumn{3}{c}{ Kolmogorov-Smirnov } & \multicolumn{2}{c}{ Shapiro-Wilk } \\
\cline { 2 - 7 } & \multicolumn{1}{c}{ Estadístico } & gl & Sig. & Estadístico & gl & Sig. \\
\hline Ancho de la Cápsula Cefálica & 0,272 & 15 & 0,004 & 0,699 & 15 & 0,000 \\
Longitud de cuernos & 0,247 & 15 & 0,014 & 0,906 & 15 & 0,119 \\
Longitud de larva & 0,153 & 15 & $0,200^{*}$ & 0,953 & 15 & 0,567 \\
\hline *. Esto es un límite inferior de la significación verdadera. & & & & & \\
a. Corrección de significación de Lilliefors & & & & & &
\end{tabular}

En la Tabla 11, se observa la prueba de normalidad del estadístico Shapiro-Wilk, puesto que los datos son menores a 50, en ella se observa que el p. valor o "Sig" es mayor a 0,01 en lo que se refiere longitud de cuernos y longitud de larvas, estos datos siguen una distribución normal, pudiendo hacer una prueba paramétrica, en cambio el ancho de la cápsula cefálica, el p. valor es menor a 0,05 indicándonos que los datos no tienen una distribución normal y por consiguiente no se puede hacer una prueba paramétrica.

\section{Tabla 12}

Regresión Lineal Simple para longitud de cuernos y longitud de la larva (mm) Estadio IV Resumen del modelo

\begin{tabular}{rrrrrr}
\hline Modelo & R & R cuadrado & $\begin{array}{c}\text { R cuadrado } \\
\text { ajustado }\end{array}$ & $\begin{array}{c}\text { Error estándar de } \\
\text { la estimación }\end{array}$ \\
\hline 1 & $0,225 \mathrm{a}$ & 0,051 & $-0,022$ & 0,59102 \\
\hline
\end{tabular}

a. Predictores: (Constante), Longitud de larva

En la Tabla 12, se observa el R2 o Coeficiente de Determinación, que expresa la varianza de la variable dependiente que esta explicada por la variable independiente, en este cuadro se observa que tiene un valor de 5,1\% de la variación de la longitud del cuerno esta explicada por la longitud de larva, así mismo se muestra el Coeficiente de Correlación de Pearson con 22,5\% existiendo una correlación no significativa para las variables en estudio.

Tabla 13

ANVA de Regresión Lineal Simple para la longitud del cuerno y longitud de la larva (mm) Estadio IV ANVA $^{\mathbf{a}}$

\begin{tabular}{lllrrrr}
\hline \multicolumn{1}{c}{ Modelo } & \multicolumn{2}{c}{$\begin{array}{c}\text { Suma de } \\
\text { cuadrados }\end{array}$} & gl & Media cuadrática & F & Sig. \\
\hline 1 & Regresión & 0,243 & 1 & 0,243 & 0,696 & 0,4 \\
& Residuo & 4,541 & 13 & 0,349 & & \\
& Total & 4,784 & 14 & & & \\
\hline
\end{tabular}

a. Variable dependiente: Longitud de cuernos

b. Predicciones: (Constante), longitud de larva

En la Tabla 13 se observa que $\mathrm{p}$. valor $>0,05$ lo que indica que no es posible construir un modelo de regresión a partir de estas dos variables, por lo que se concluye que las variables no están linealmente relacionadas. 


\section{Tabla 14}

Prueba de Nornalidad para ancho de la cápsula cefálica, longitud de los cuernos y longitud de la larva (mm) Estadio $V$

Pruebas de normalidad

\begin{tabular}{lrrrrrr}
\hline & \multicolumn{3}{c}{ Kolmogorov-Smirnov ${ }^{\mathbf{a}}$} & \multicolumn{3}{c}{ Shapiro-Wilk } \\
\cline { 2 - 8 } & Estadístico & gl & Sig. & Estadístico & gl & \multicolumn{1}{c}{ Sig. } \\
\hline Ancho de la Cápsula Cefálica & 0,191 & 15 & 0,147 & 0,914 & 15 & 0,155 \\
Longitud de cuernos & 0,210 & 15 & 0,074 & 0,908 & 15 & 0,127 \\
Longitud de larva & 0,269 & 15 & 0,005 & 0,827 & 15 & 0,008 \\
\hline
\end{tabular}

a. Corrección de significación de Lilliefors

En la Tabla 14, se observa la prueba de normalidad del estadístico Shapiro-Wilk, ya que los datos son menores a 50, en ella se observa que el p. valor o "Sig" es mayor a 0,01 en lo que se refiere al ancho de la cápsula cefálica y longitud de cuernos, estos datos siguen una distribución normal, pudiendo hacer una prueba paramétrica, en cambio longitud de larvas, se observa que el p. valor es menor a 0,05 lo cual nos dice que no tiene una distribución normal y por consiguiente no se puede hacer una prueba paramétrica.

Tabla 15

Regresión Lineal Simple para Ancho de cápsula cefálica y longitud de cuernos (mm) Estadio V Resumen del modelo

\begin{tabular}{lccccc}
\hline Modelo & R & & R cuadrado & $\begin{array}{c}\text { R cuadrado } \\
\text { ajustado }\end{array}$ & $\begin{array}{c}\text { Error estándar de } \\
\text { la estimación }\end{array}$ \\
\hline 1 & & $0,133^{\text {a }}$ & 0,018 & $-0,058$ & 1,16406 \\
\hline
\end{tabular}

a. Predictores: (Constante), Ancho de la Cápsula Cefálica

En la Tabla 15, se observa el R2 o Coeficiente de Determinación, que expresa la varianza de la variable dependiente que esta explicada por la variable independiente, en este cuadro se observa que tiene un valor de 1,8\% de la variación de la longitud del cuerno esta explicada por el ancho de la cápsula cefálica, así mismo se muestra el Coeficiente de Correlación de Pearson con 13,3\% existiendo una correlación no significativa para las variables en estudio.

Tabla 16

ANVA de Regresión Lineal Simple para la longitud del cuerno y longitud de la larva (mm) Estadio V ANVA $^{\mathrm{a}}$

\begin{tabular}{llrrrrr}
\hline Modelo & & $\begin{array}{c}\text { Suma de } \\
\text { cuadrados }\end{array}$ & gl & cuadrática & F & Sig. \\
\hline 1 & Regresión & 0,318 & 1 & 0,318 & 0,235 & $0,636^{\mathrm{b}}$ \\
& Residuo & 17,615 & 13 & 1,355 & & \\
Total & 17,933 & 14 & & & \\
\multicolumn{5}{c}{} \\
\hline a. Variable dependiente: Longitud de cuernos \\
b. Predictores: (Constante), Ancho de la Cápsula Cefálica
\end{tabular}

En la Tabla 16 se observa que p. valor > 0,05 lo que indica que no es posible construir un modelo de regresión a partir de estas dos variables, por lo que se concluye que las variables no están linealmente relacionadas. 


\section{Conclusiones}

Caligo idomeneus (Wilson, 1992), (Buttreffy y Planets, 2016), es una mariposa que presenta cinco estadios larvales y, al conocer su ciclo biológico, permitirá criar intensivamente y con facilidad en condiciones de mariposario, por lo que posee un buen potencial para la producción comercial. Los únicos sustratos de alimentación fueron Heliconius y Musa. Heliconia se reproduce fácilmente por lo que es un excelente sustrato de alimentación (Bart, 1991).

Se desarrolló una metodología adecuada para la reproducción tanto en laboratorio como en el mariposario, logrando un impacto favorable para la posterior producción masal. 


\section{Referencias bibliográficas}

ANDRADE, C. (2000). Biodiversidad y conservación de la fauna Colombiana en memorias I Congreso Colombiano de Zoología. Instituto de Ciencias Naturales- Universidad Nacional de Colombia.

ANDREWS L. Y RUTILO Q. (1989). Manejo integrado de plagas insectiles en la agricultura. Estado actual y futuro. Departamento de Protección vegetal-escuela. Agrícola Panamericana El Zamorano, Honduras.

ASTO, C. y GUITIERREZ, M. (2014), Implementación de mariposarios como atractivo turístico en el departamento de Loreto, Perú.

BARTH, F. (1991). Insects and Flowers. The biology of partnership. Princenton University Press. 406 pp.

BROWN, K.S., TRIGO, Jr. (1991). Conservation of neotropical paleoenvironments: Insects as indicators. In: Collins, N. M and J.A. Thomas(Eds), Conservation of Insects and their hábitats. Press, London, pp. 349-404.

BUTTREFLY PLANETS, 2016. https:// www. butterflyplanet. com/ brassolidae- caligoidomeneus-idomenides- pair-a1- pe.html

CANTARERO K., CANALES O., MENDOZA A. Y MARTÍNEZ L. (2012). Ciclo de vida de las especies Caligo memno (Lepidoptera: Brassolinae) y Heliconius ismenius (Lepidoptera: Heliconinae) bajo condiciones controladas.

CATALOGUE OF LIFE: 2019. Caligo idomeneus Linnaeus, 1758 in Species 2000 \& ITIS, Catalogue of Life.

CONSTANTINO, L. (1996). Ciclos de vida y plantas hospederas de lepidópteros diurnos con potencial económico en condiciones de Colinas bajas del Choco biogeográfico. II Seminario. Investigación y manejo de fauna para la construcción de sistemas sostenibles. Instituto Alexander Von Humboldt Cali, Marzo 28-30 de 1996. Memorias, pp 75-86

DE VRIES, P. (1987). The butterflyes of Costa Rica and their natural history. Princenton University Press, New Jersey. 327 pp.

FLORES J. Y GIL J. (2021). Ciclo biológico del gusano cabrito del plátano (Caligo teucer semicaerulea Joicey \& Kaye, 1917) en Tingo María, Perú

GILBERT, L. (1975). Ecological consequences of coevolved mutualism between butterflies and plantas. Pp 210-240. En: L. E GILBERT y P. H. RAVEN Coevolution of aninals and plants. 


\section{LUCCI- FREITAS A., BENSON W., MARINI-FILHO O. Y MAMERI DE CARVALHO R.}

2016. Territoriality by the dawn's early light: the Neotropical butterfly Caligo idomeneus (Nymphalidae: Brassolinae).

MASO, A y PIJOAN (1997). Manual observar mariposas. Barcelona, editorial planeta 317 pp.

MULANOVICH A. (2007). Guía para el manejo sustentable de mariposas en el Perú. Disponible en: http://www.siamazonia.org.pe/archivos/publicaciones/L032.pdf.

OWL BUTTERFLY, 2016. https:// www. anywhere. com/ flora-fauna/ invertebrates/ owlbutterfly

PROMPEX, IIAP Y GTZ. (2007). Guía para el manejo sustentable de mariposas en el Perú.

RAMIREZ, J. A. (1994). Manejo de fauna Silvestre y los limites previsibles de la sustentabilidad. Seminario. Investigación y manejo de fauna para el desarrollo de sistemas sostenibles de producción en el trópico. CIPAV, IMCA, U. Javeriana. Buga, marzo 10-12 Memorias.

SANCHEZ, L. (2004). Protocolo de cría para dos especies de mariposas, Ascia monuste y Leptophobia aripa (Lepidoptera: Pieridae) bajo condiciones controladas en el Municipio de la Mesa, Cundinamarca.

WILSON, E. (1992). The Diversity of Life. New York: W. W. Norton \& Co. 


\section{Anexos}

Anexo 1. Ubicación del mariposario

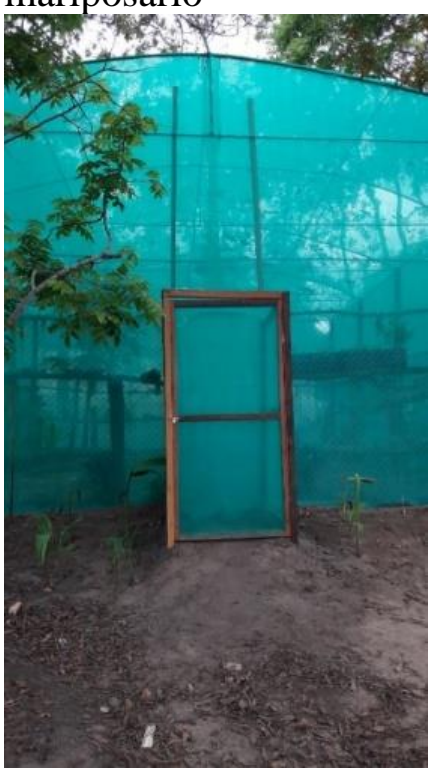

Fuente: Doria, 2018.

\section{Anexo 2. Posturas de Caligo idomeneus}

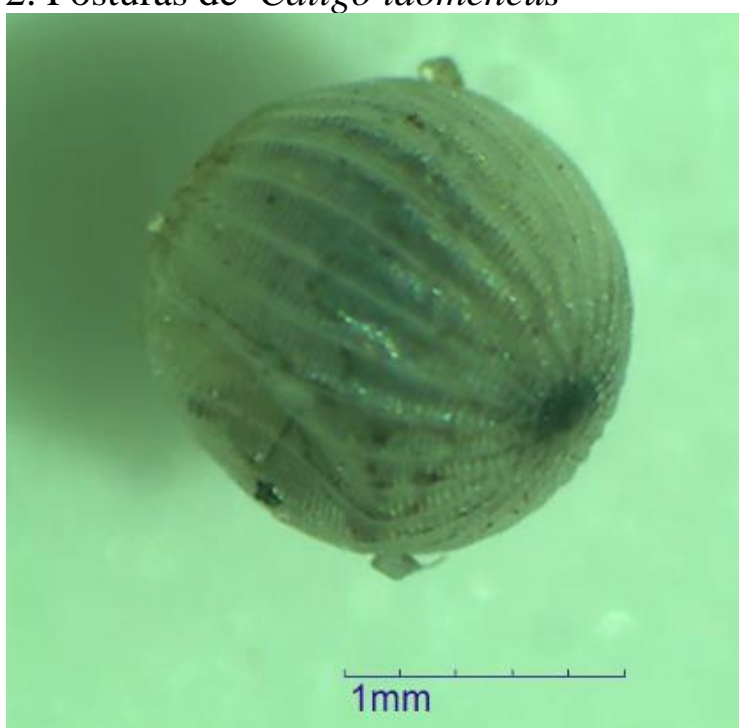

Fuente: Doria, 2018.
Anexo 3. Larvas de Caligo idomeneus

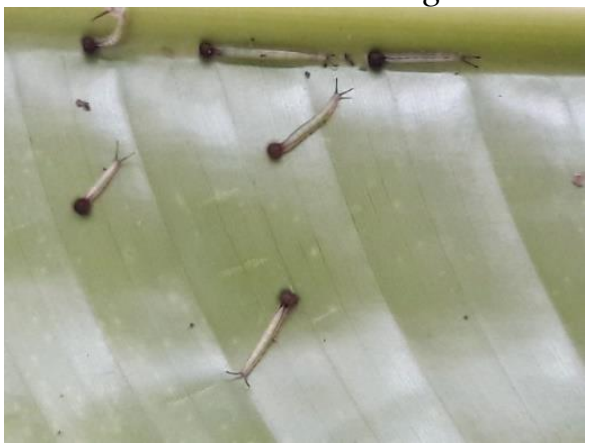

Fuente: Doria, 2018.
Anexo 4. Adultos de Caligo idomeneus

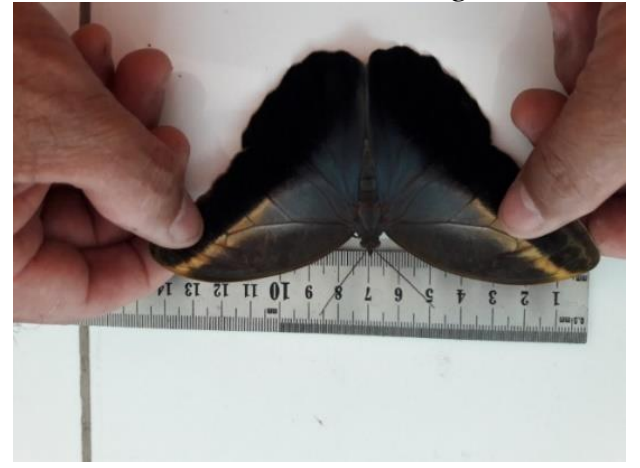

Fuente: Doria, 2018.

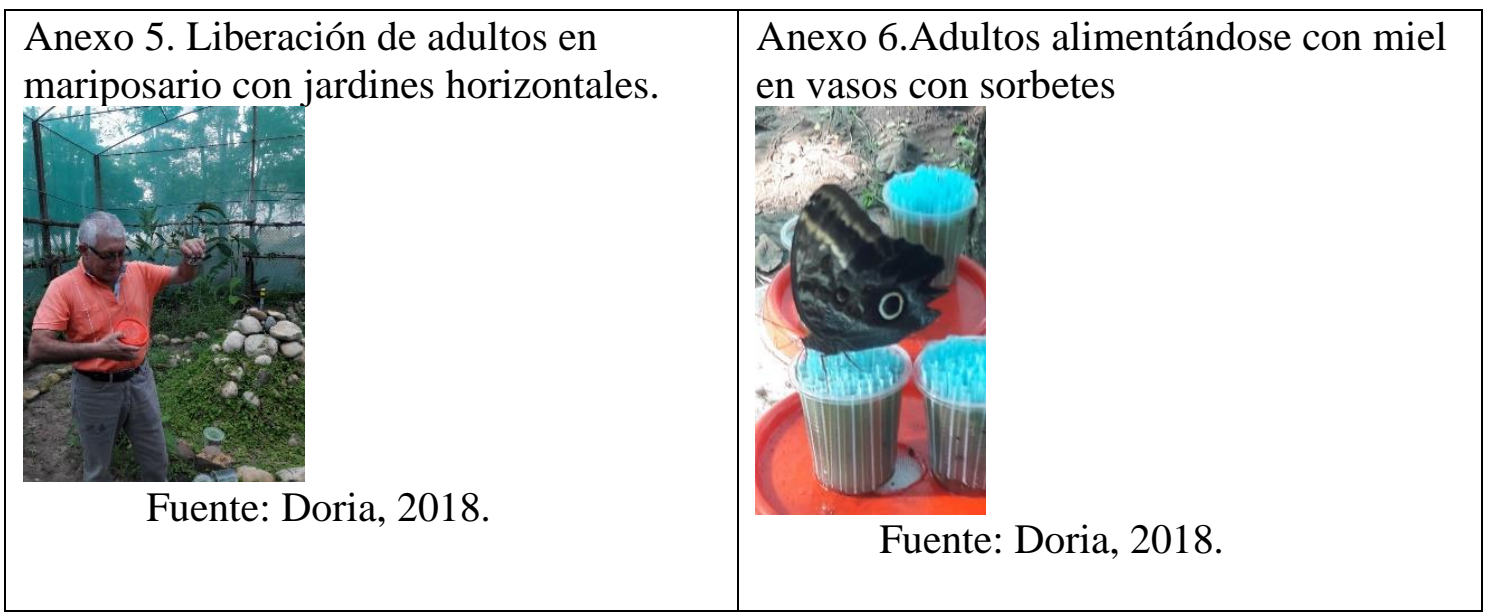

Citar como: Doria Bolaños, M., Ramirez Navarro, M., \& Fachin Ruiz, G. (2021). Ciclo biológico de Caligo Idomeneus (Nymphalidae) bajo condiciones controladas con jardines verticales en Tarapoto. Revista Agrotecnológica Amazónica, 1(1), 20-33. https://doi.org/10.51252/raa.v1i1.105 


\section{Conflicto de intereses}

Ninguno.

\section{Contribuciones de los autores}

Todos con la misma participación 